\title{
La carga inmediata de implantes transicionales en sobredentaduras mandibulares en adultos mayores
}

\author{
VELASCO ORTEGA E* \\ SEGURA EGEA JJ** \\ LINARES GANCEDO $\mathbf{D} * * *$ \\ MEDEL SOTERAS R** \\ POYATO FERRERA $\mathbf{M} * * *$
}

\begin{abstract}
Velasco Ortega E, Segura Egea JJ, Linares Gancedo D, Medel Soteras R, Poyato Ferrera M. La carga inmediata de implantes transicionales en sobredentaduras mandibulares en adultos mayores. Av Periodon Implantol. 2004; 16, 2: 107-113.
\end{abstract}

\begin{abstract}
RESUIMEN
Introducción. La implantología oral constituye una importante modalidad terapéutica en el tratamiento prostodóncico de los pacientes con pérdida dental total. El estudio muestra la evaluación de los pacientes edéntulos totales tratados con sobredentaduras mandibulares mediante la carga inmediata de los mini implantes de titanio con superficie grabada con ácidos. Métodos. 18 pacientes edéntulos totales fueron tratados con 72 mini implantes transicionales Sendax en la mandíbula para su rehabilitación prostodóncica con sobredentaduras. Todos los implantes fueron insertados en l cirugía y fueron cargados inmediatamente en la misma sesión. Los hallazgos clínicos (implantológicos y prostodóncicos) se han seguido al menos durante 12 meses. Resultados. Después de un seguimiento clínico medio de 20,8 meses (rango: 12-48 meses), los resultados indican un éxito de los implantes del $97,3 \%$ porque 2 implantes fracasaron. El 100\% de los pacientes fueron tratados con una sobredentadura implantosoportada con retenedores de bolas sobre 4 implantes. Conclusiones. Los resultados del presente estudio indican que la rehabilitación prostodóncica de la mandíbula con sobredentaduras implantosoportadas de los pacientes edéntulos mediante la carga inmediata de implantes transicionales de titanio pueden obtener la oseointegración y representar una terapéutica odontológica con éxito.
\end{abstract}

\section{PALABRAS CLAVE}

Implantes transicionales, carga inmediata, sobredentaduras, superficie grabada con ácidos, oseointegración, implantología oral.

Aceptado para publicación: Marzo 2004

\section{INTRODUCCIÓN}

El tratamiento con prótesis completa puede representar una opción terapéutica para restaurar la función y la estética del paciente desdentado total. Sin embargo, las prótesis completa pueden crear problemas funcionales y psicológicos importantes como consecuencia de una mala adaptación (1).

\footnotetext{
* Profesor Titular de Odontología Integrada de Adultos y Gerodontología. Facultad de Odontología. Universidad de Sevilla.

** Profesor Asociado de Odontología Integrada de Adultos. Facultad de Odontología. Universidad de Sevilla.

*** Profesor Colaborador Docente de Odontología Integrada de Adultos y de Gerodontología. Facultad de Odontología. Universidad de Sevilla.
} 
El tratamiento con implantes dentales y el descubrimiento de los fenómenos biológicos de la oseointegración han revolucionado la terapéutica de los pacientes con edentulismo total (2). La rehabilitación fija implantosoportada del paciente desdentado total puede no estar indicada por problemas anatómicos, funcionales o económicos. En estos casos, la posibilidad de realizar una sobredentadura mediante la inserción de varios implantes en el paciente desdentado inferior representa, en la actualidad una terapéutica muy favorable con un éxito superior al $90 \%$ (3-6).

Generalmente, se ha aceptado en implantología oral que la oseointegración requiere un periodo de cicatrización libre de carga funcional de al menos 3 meses en la mandíbula y de 5-6 meses en el maxilar superior, ya que se ha postulado que la carga prematura podía provocar una encapsulación fibrosa que impidiera la conexión directa entre el hueso y la superficie del implante $(7,8)$. Sin embargo, actualmente se considera que los protocolos prostodóncicos implantológicos con carga precoz e inmediata per se no impiden la oseointegración, siempre que se controle los micromovimientos durante la cicatrización y se haga una selección cuidadosa de los pacientes con buena calidad y cantidad de hueso y una mejor estabilidad primaria de los implantes $(9,10)$.

Otro aspecto importante relacionado con la carga inmediata de los implantes es el desarrollo de los implantes con un diseño que favorece su inserción quirúrgica y su conexión con los pilares mejorando los resultados prostodóncicos (11-13). Además, el tratamiento de la superficie mediante procedimientos químicos (ej. grabado ácido, chorreado de arena) sugieren una mayor y más rápida unión del hueso alveolar al implante que puede también mejorar la carga funcional inmediata $(14,15)$.

Los protocolos de carga inmediata en la mandíbula de pacientes edéntulos han sido introducidos con un éxito similar a los estudios con carga diferida (16-19). En este sentido, un estudio retrospectivo europeo demuestra un éxito del $96,9 \%$ en 226 pacientes que fueron tratados con 4 implantes insertados en el sector anterior mandibular y cargados inmediatamente con sobredentaduras retenidas con barras con un seguimiento medio de 6,4 años (16).

Los implantes transicionales se han incorporado a la práctica odontológica para soportar de forma inmediata y provisional una prótesis removible mientras los implantes definitivos consiguen la oseointegración. Cuando los implantes definitivos pueden soportar la rehabilitación prostodóncica, los implantes provisionales o transicionales son extraídos. Recientemente, se ha demostrado que los implantes transicionales pueden oseointegrarse y ofrecer una buena fijación con el hueso alveolar que se incrementa con el tiempo prolongado de carga funcional $(20,21)$.

El objetivo del presente estudio era la valoración clínica del tratamiento con sobredentaduras mandibulares mediante la inserción de implantes transicionales con carga inmediata en pacientes edéntulos totales.

\section{PACIENTES Y MÉTODOS}

El presente estudio ha sido realizado por profesores de las unidades docentes de Odontología Integrada de Adultos y de Gerodontología de la Facultad de Odontología de la Universidad de Sevilla.

Previamente a la realización del estudio, aquellos pacientes que padecían trastornos sistémicos graves que podían comprometer la oseointegración fueron excluidos del estudio (22). Todos los pacientes seleccionados eran adultos mayores de ambos sexos y edéntulos totales (Figura 1).

Todos los pacientes seleccionados en el estudio fueron informados del protocolo del tratamiento con implantes, de los aspectos quirúrgicos y prostodóncicos, así como de la posible existencia de complicaciones y pérdida de implantes. Los pacientes autorizaron el tratamiento implantológico mediante un consentimiento informado. Antes del tratamiento, todos los pacientes fueron evaluados radiológicamente, con una ortopantomografía, y en los casos de reabsorción mandibular extrema con una tomografía axial computarizada.

CIRUGÍA. Una hora antes de la cirugía, los pacientes comenzaron un régimen antibiótico preventivo (amoxicilina + clavulánico) durante una semana. Todos los pacientes recibieron anestesia local. Un total de 4

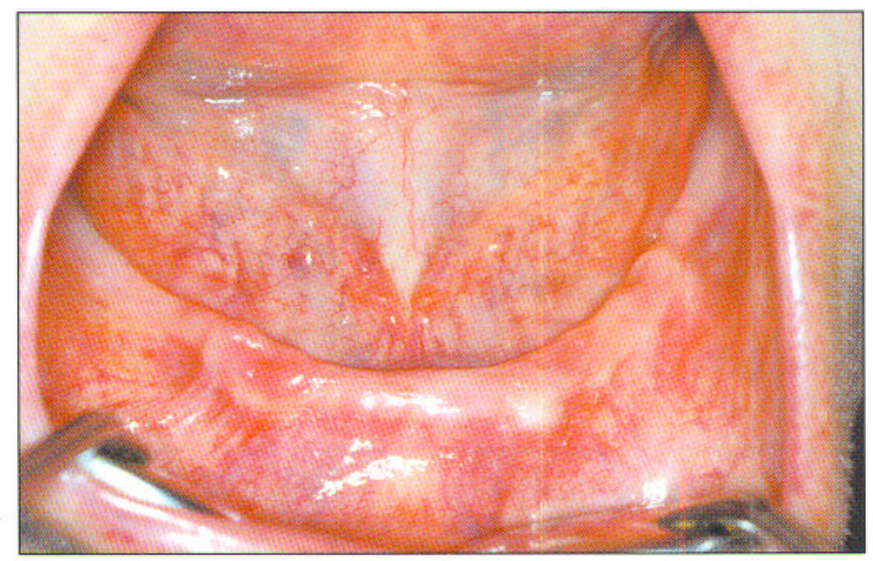

Fig 1: Aspecto oral del paciente edéntulo. 


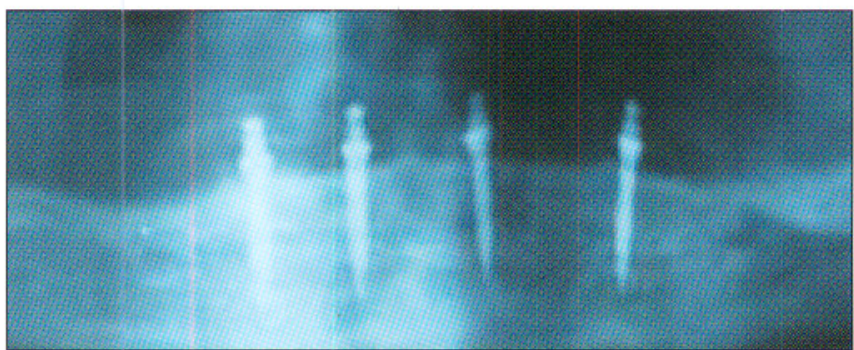

Fig 2: Ortopantomografía inmediata después de la inserción de los 4 implantes.

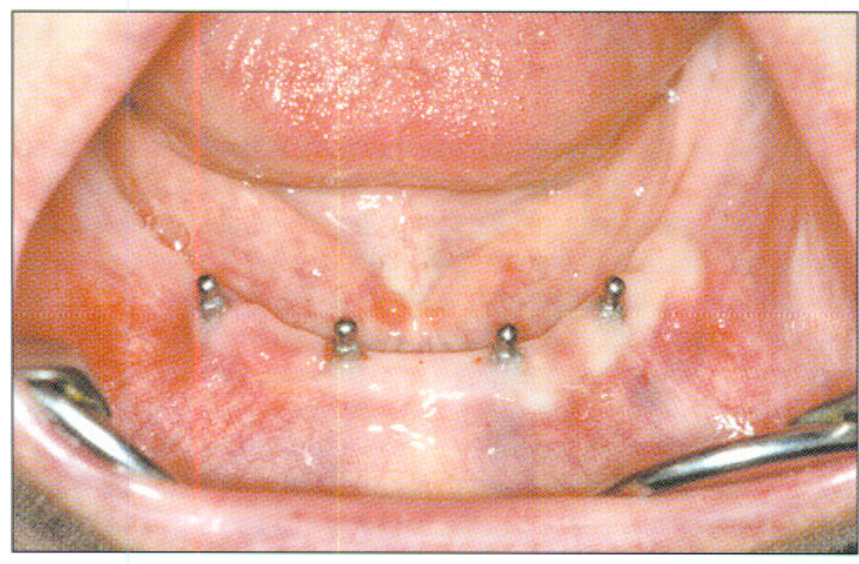

Fig 3: Aspecto clínico de los implantes insertados sin incisión quirúrgica en la mandíbula.

implantes Sendax ${ }^{\circledast}$ (IMTEC, Arhmore, EE.UU.) (Figura 2) con un retenedor de bola en la cabeza, roscados de $1,8 \mathrm{~mm}$ de diámetro y $13 \mathrm{~mm}$ de longitud, fueron insertados en cada paciente entre los agujeros mentonianos y todos fueron estables después de la inserción (Figura 3). El procedimiento quirúrgico básico no exigió ningún tipo de incisión en la mandíbula edéntula. La preparación del lecho y la inserción de los implantes se realizó según el protocolo estandarizado con 1 fresa de $1,1 \mathrm{~mm}$ de diámetro, a una velocidad constante de 800 r.p.m. A los pacientes se les recomendó el enjuague diario con clorhexidina durante los primeros 30 días.

PROSTODONCIA. Todos los pacientes eran portadores de una prótesis completa. En el mismo acto odontológico, después de la inserción de los implantes se tomó la impresión inferior y se incorporaron los retenedores de bola en la parte interna de la prótesis completa mandibular. Posteriormente se realizó la carga inmediata funcional de los implantes mediante la colocación de una sobredentadura (Figura 4). El tiempo transcurrido de seguimiento clínico desde la carga funcional de los implantes fué al menos de 1 año.

ANÁLISIS ESTADÍSTICO. Se ha realizado una estadística descriptiva de los hallazgos clínicos del estudio,

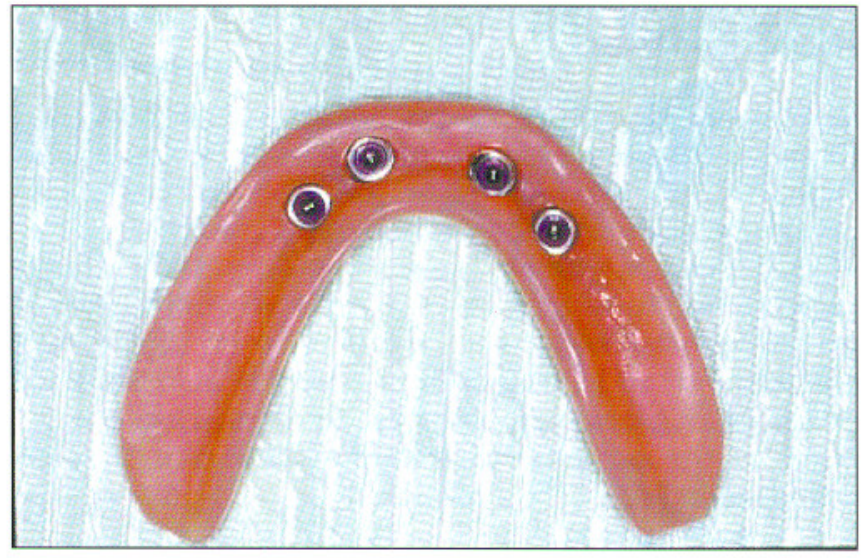

Fig 4: Aspecto clínico de la sobredentadura mandibular con los retenedores de bolas.

con referencia a las variables demográficas de los pacientes, los implantes insertados, las restauraciones prostodóncicas realizadas, así como el éxito y las complicaciones del tratamiento.

\section{RESULTADOS}

PACIENTES. 18 pacientes edéntulos totales portadores de una prótesis completa (6 hombres y 12 mujeres) participaron en el estudio, con una edad media de 68,05 años (rango: 63-82 años).

IMPLANTES. Se insertaron un total de implantes 72 implantes Sendax ${ }^{\circledast}$ en la mandíbula edéntula, de los correspondientes 18 pacientes, lo que representa una media de 4 implantes por paciente. Con respecto a la longitud, todos los implantes eran de $13 \mathrm{~mm}$. El 100\% de los implantes fueron insertados de forma diferida, tras un periodo mínimo de 6 meses después de la última extracción dental.

Se perdieron un total de 2 implantes en 2 pacientes que después de 45 días se sustituyeron por otros 2 implantes Sendax ${ }^{\otimes}$ lo que supone un índice de éxito del $97,3 \%$. Durante el seguimiento clínico no ha habido ninguna otra complicación.

PROSTODONCIA. En todos los pacientes se realizó la carga funcional inmediata de los implantes en la misma sesión mediante una sobredentadura con los 4 retenedores para bolas. En este sentido, un total de 18 prótesis completas inferiores fueron transformadas en sobredentaduras implantorretenidas.

El seguimiento clínico medio de los pacientes estudiados ha sido de 20,8 meses (rango: 12 meses-48 meses) 


\section{DISCUSIÓN}

En la actualidad, la carga inmediata de los implantes dentales constituye un reto permanente de una modalidad terapéutica en auge en el tratamiento del pacientes con edentulismo total. En este sentido, a través de un correcto diagnóstico y una cuidada planificación, mediante un estricto protocolo quirúrgico y prostodóncico se puede mejorar la situación del paciente evitando los inconvenientes, la incomodidad y los problemas psicológicos relacionados con el periodo de espera del tratamiento implantológico oral clásico (23).

La carga inmediata en implantología oral se ha realizado en pacientes con pérdidas dentales unitarias (24), en pacientes parcialmente desdentados (25), pero sobre todo, en el tratamiento implantológico de pacientes edéntulos totales, especialmente en la mandíbula (16-19, 26-30). En estos casos, los protocolos han consistido fundamentalmente, en la ferulización de varios implantes insertados (4-10 implantes) mediante la colocación inmediata de una prótesis fija atornillada $(19,26,27)$ o de una sobredentadura (16, 29).

Los resultados de estos estudios de carga inmediata en el tratamiento con sobredentaduras mandibulares han demostrado unos altos índices de éxito $(29,31$, 32). Gatti y cols. (29) valoran los hallazgos de sobredentaduras retenidas mediante barras con 4 implantes con carga inmediata, seguidos durante una media de 37 meses (rango 25-60 meses) con un éxito del $96 \%$. Así mismo, se han comparado el éxito de sobredentaduras en la mandíbula con carga funcional inmediata y tardía $(31,32)$. En este sentido, Chiapasco y cols (31) han comparado los resultados clínicos (a los 6, 12 y 24 meses) de implantes Branemark cargados de forma inmediata y tardía en 2 grupos de pacientes que fueron tratados con sobredentaduras en la mandíbula sobre 4 implantes con un éxito similar en ambos grupos del 97,5\%. Romeo y cols (32) también comparan los hallazgos clínicos de sobredentaduras en la mandíbula con 4 implantes ITI en 2 grupos de pacientes con carga funcional inmediata y tardía, con un éxito del $100 \%$ y del $97,5 \%$, respectivamente, a los 24 meses.

Los implantes transicionales se han utilizado para soportar temporalmente restauraciones prostodóncicas fijas o removibles durante el periodo libre de carga funcional en los implantes definitivos $(33,34)$. En este sentido, los implantes transicionales son cargados inmediatamente y el grado de oseointegración de estos implantes es alto según se ha demostrado histológicamente, sobre todo en la mandíbula donde se necesita una fuerza de torque para su remoción más elevada $(20,21)$. Además, la experiencia demues- tra que estos implantes transicionales están más oseointegrados conforme se incrementa el tiempo transcurrido desde su inserción lo que puede ser una indicación para aplicaciones clínicas a largo plazo (21).

Los resultados de este estudio indican que la carga inmediata de implantes orales transicionales pueden conseguir la oseointegración adecuada y constituir una técnica predecible y con éxito en el tratamiento con sobredentaduras mandibulares en los pacientes edéntulos totales. En este sentido, siguiendo un protocolo implantológico muy sencillo desde un punto de vista quirúrgico y prostodóncico se ha obtenido una supervivencia y éxito de los implantes de $97,3 \%$.

En el presente protocolo, la inserción de los implantes se realiza sin incisión quirúrgica y exposición del hueso subyacente ni sutura, por lo que el procedimiento quirúrgico se simplifica bastante, y las molestias para el paciente son escasas. En este sentido, se trata de una técnica minimamente invasiva que solamente requiere la destreza y práctica implantológica por parte del profesional. Desde esta perspectiva, se confirma que la inserción no sumergida de los implantes es una técnica predecible y proporciona una buena respuesta tisular ósea y de los tejidos blandos periimplantarios, aún en los protocolos de carga inmediata $(10,35)$.

El tratamiento con implantes orales puede presentar complicaciones quirúrgicas y prostodóncicas, que, a veces, provocan el fracaso de esta terapéutica (36). Las pérdidas de los implantes pueden ser consecuencia de complicaciones precoces (fracaso en conseguir la oseointegración) y tardías (fracaso en mantener la oseointegración. Los fracasos precoces puede ser originados por factores relacionados con el procedimiento quirúrgico implantológico (ej. sobrecalentamiento del lecho implantario, falta de estabilidad primaria) que impiden una adecuada unión entre la superficie del implante y el hueso) (37). Los fracasos tardíos están más relacionados con la restauración prostodóncica (ej. excesiva carga funcional y/o un incorrecto diseño) y la perdida de soporte periimplantario (ej. periimplantitis) (36-38). En el presente estudio, un total de 2 implantes presentaron movilidad al mes de su seguimiento clínico y fueron extraídos, aunque posteriormente fueron reemplazados.

Los implantes utilizados en el presente estudio son implantes roscados con superficie grabada con ácidos que representa un aspecto muy importante en la implantología actual. En efecto, se ha demostrado que el tratamiento de la superficie de los implantes dentales, incrementando su rugosidad puede favorecer la unión al tejido óseo mejorando la oseointegración $(14,15)$ y reduciendo el tiempo de espera de la carga funcional prostodóncica en pacientes con pérdidas 
dentales $(39,40)$, como lo demuestra un estudio realizado con sobredentaduras mandibulares sobre implantes con superficie grabada con ácidos y cargados a las 6 semanas de su inserción con ún exito del $97,8 \%$ a 1 año de seguimiento clínico (41).

Un objetivo esencial del presente estudio era la carga inmediata de los implantes con sobredentaduras mandibulares. En este sentido, la fase prostodóncica fué realizada de forma inmediata en la misma sesión clínica después de la inserción de los implantes. Se tomaron impresiones de los pacientes con los implantes insertados, se realizaron los modelos correspondientes y por un método indirecto -mediante los análogos de implantes- se realizaron los rebasados de la prótesis completa inferior con la incorporación de los ataches para bolas (Figura 4).

En el presente estudio se han realizado las sobredentaduras mandibulares sobre 4 implantes en pacientes, edéntulos totales durante mucho tiempo, que por sus características orales con una cresta alveolar muy estrecha necesitaban la utilización de técnicas más complejas (ej. injertos óseos) para su rehabilitación con prótesis sobre implantes. En este sentido, el método utilizado con 4 implantes de diámetro muy reducido (1,8 mm de diámetro) con el extremo superior en forma de bola, permite una retención mecánica muy favorable en sobredentaduras mandibulares y puede constituir una terapéutica favorable, sencilla y económica.

\section{CONCLUSIONES}

La implantología oral ha revolucionado el tratamiento de los pacientes mayores con edentulismo total. En efecto, como demuestra los resultados clínicos del presente estudio, los protocolos con carga inmediata de implantes y la rehabilitación mediante sobredentaduras mandibulares pueden mejorar los inconvenientes de la prótesis completa convencional, y transformar de forma inmediata la salud oral de los pacientes adultos de edad avanzada con un impacto positivo en su calidad de vida.

\section{ABSTRAC}

Introduction. Oral implantology constitute an important therapeutic modality in the prosthodontic treatment of patients with total tooth loss. This study reports the evaluation of edentulous patients treated with overdentures in the mandible by immediate loa- ding of titanium miniimplants with acid-etched surface.

Patients and Methods. 18 edentulous patients were treated with 72 Sendax $^{\circledR}$ transitional mini implants for prosthodontic rehabilitation with overdentures in the mandible. All implants were inserted in one-stage and were loaded immediately. Clinical findings (implant and prosthodontics) were followed during 12 months at least.

Results. After a mean follow-up of 20.8 months (range: 12-48 months), clinical results indicate a success rate of implants of $97,3 \%$. Two implants were lost. $100 \%$ of patients were treated with overdentures with ball attachments retained with 4 implants.

Conclusions. Clinical results of this study indicate that prosthodontic rehabilitation in the mandible of edentulous patients with overdentures supported by transitional titanium implants can achieve osseointegration and were loaded immediately as a successful dental treatment.

\section{KEY WORDS}

Transitional implants, immediate loading, overdentures, acid-etched surface, osseointegration, implant dentistry.

\section{CORRESPONDENCIA}

Dr. Eugenio Velasco Ortega

C/ José Laguillo n ${ }^{\circ} 18,2^{\circ}-6$

Tfno: 954421805 e-mail: evelasco@us.es

41003 Sevilla

\section{BIBLIOGRAFÍA}

1. Carlsson GE. Clinical morbidity and sequelae of treatment with complete dentures. J Prosthet dent 1997; 79: 17-23.

2. Worthington P, Lang BR, Rubestein JE. Osseointegration in Dentistry. An overview. (2 ${ }^{\mathrm{a}}$ ed.). Quintessence: Chicago. 2003.

3. Batenburg RHK, Meijer HJA, Raghoebar GM, Vissink A. Treatment concept for mandibular overdentures supported by endosseous implants: aliterature review. Int $\mathrm{J}$ Oral Maxillofac Implants 1998; 13: 539-45. 
4. Bergendal T, Engquist B. Implant-supported overdentures: a longitudinal prospective study. Int J Oral Maxillofac Implants 1998; 13: 253-62.

5. Sadowsky SJ. Mandibular implant-retained overdentures: a literature review. J Prosthet Dent 2001; 86: 468-73.

6. Feine JS, Carlsson GE. Implant overdentures. The standard of care for edentulous patients. Quintessence: Chicago. 2003.

7. Branemark PI, Hansson BO, Adell R, et al. Osseointegrated implants in the treatment of the edentulous jaws. Experience from a 10-year period. Scand J Plast Reconstr Surg 1977; 16: 1-132.

8. Branemark PI, Zarb GA, Albrektsson T (eds.). Tissue integrated prostheses: osseointegration in clinical dentistry. Quintessence: Chicago. 1985.

9. Szmukler-Moncler S, Piatelli A, Favero GA, Dubruille JH. Considerations preliminary to the application of early and immediate loading protocols in dental implantology. Clin Oral Impl Res 2000; 11: 12-25.

10. Meyer U, Wiesmann HP, Fillies T, Joos U. Early tissue reaction at the interface of immediately loaded dental implants. Int J Oral Maxillofac Implants 2003; 18: 489-99.

11. Sykaras N, lacopino M, Marker VA, Triplett RG, Woody RD. Implant materials, designs, and surface topographies: their effect on osseointegration. A literature review. Int J Oral Maxillofac Implants 2000; 15: 675-90.

12. Binon PP. Implants and components: entering the new millennium. Int J Oral Maxillofac Implants 2000; 15: 76-94.

13. Marinho VC, Celleti R, Bracchetti G, Petrone G Minkin C, Piatelli A. Sanblasted and acid-etched dental implants: a histologic study in rats. Int J Oral Maxillofac Implants 2003; 18: 75-81.

14. Kim YH, Koak JY, Chang IT, Wennerberg A, Heo SJ. A histomorphometric analysis of the effects of various surface treatment methods on osseointegration. Int $\mathrm{J}$ Oral Maxillofac Implants 2003; 18: 349-56.

15. Khang W, Feldman S, Hawley CE, Gunsolley J. A multicenter study comparing dual acid-etched and machines-surfaced implants in various bone qualities. J Peridontol 2001; 72: 1384-90.

16. Chiapasco M, Gatti C, Rossi E, Haefliger W, Markwalder TH. Implant-retained mandibular overdentures with immediate loading: a retrospective multicenter study on 226 consecutive cases. Clin Oral Impl Res 1997; 8: 48-57.

17. Horiuchi K, Uchida H, Yamamoto K, Sugimura M. Immediate loading of Branemark system implants following placement in edentulous patients: a clinical report. Int J Oral Maxillofac Implants 2000; 15: 8924-830.

18. Testori T, Del Fabbro M, Szmukler-Moncler S, Francetti L, Weinstein RL. Immediate occlusal loading of Osseotite implants in the completely edentulous mandible. Int J Oral Maxillofac Implants 2003; 18: 544-51.
19. Wolfinger GJ, Balshi TJ, Rangert B. Immediate functional loading of Branemark system implants in edentulous mandibles: clinical report of the results of developmental and simplified protocols. Int J Oral Maxillofac Implants 2003; 18: 250-7.

20. Proussaefs P. Histologic evaluation of an immediately loaded titanium provisional implant retrieved after functioning for 18 months: a clinical report. J Prosthet Dent 2003; 89: 331-4.

21. Simon H, Caputo AA. Removal torque of immediately loaded transitional endosseous implnts in human subjects. Int J Oral Maxillofac Implants 2002; 17: 839-45.

22. Sugerman PB, Barber MT. Patient selection for endosseous dental implants: oral and systemic considerations. Int J Oral Maxillofac Implants 2002; 17: 191-201.

23. Gapski R, Wang HL, Mascarenhas P, Lang NP. Critical review of immediate implant loading. Clin Oral Impl Res 2003; 14: 515-27.

24. Ericsson I, Nilson H, Lindh T, Nilner K, Randow K. Immediate functional loading of Branemark single tooth. An 18 months' clinical pilot follo-up study. Clin Oral Impl Res 2000; 11: 26-33.

25. Jaffin RA, Kumar A, Berman CL. Immediate loading of implants in partially and fully edentulous jaws: a series of 27 cases. J Periodontol 2000; 71: 833-8.

26. Tarnow DP, Emtiaz S, Classi A. Immediate loading of threated implantsa at stage 1 surgery in edentulous arches: ten consecutive case reports with 1-to 5-year data. Int J Oral Maxillofac Implants 1997; 12: 319-24.

27. Schnitman PA, Wöhrle PS, Rubenstein JE, DaSilva JD, Wang $\mathrm{NH}$. Ten-year results for Branemark implants immdiatley loaded with fixed prostheses at implant placement. Int $\mathrm{J}$ Oral Maxillofac Implants 1997; 12: 495-503.

28. Randow K, Ericcson I, Nilner K, Petersson A, Glantz PO. Immediate functional loading of Branemark dental implants. A 18 month clinical follow-up study. Clin Oral Impl Res 1999; 10: 8-15.

29. Gatti C, Haefliger W, Chiapasco M. Implant-retained mandibular overdentures with immediate ioading: a prospective study of ITI implants. Int J Oral Maxillofac Implants 2000; 15: 383-8.

30. Branemark PI, Engstrand P, Ohrnell LO, Grondahl K, Nilsson P, Hagberg K, Darle C, Lekholm U. Branemark Novum: a new treatment concept for rehabilitation of the edentulous mandible. Preliminary results drom a prospective clinical follow-up study. Clin Impl Dent Rel Res 1999; 1: 2-16.

31. Chiapasco M, Abati S, Romeo E, Vogel G. Implant-retained mandibular overdentures with Branemark system MKII implants: a prospective comparative study between delayed and immediate loading. Int J Oral Maxillofac Implants 2001; 16: $537-46$.

32. Romeo E, Chiapasco M, Lazza A, Casentini P, Ghisolfi M, lorio M, Vogel G. Implant-retained mandibular overdentures with ITI implants. A comparison of 2-year results between delayed and immediate loading. Clin Oral Impl Res 2002; 13: 495501. 
33. Bohsali K, Simon H, Kan JY, Redd M. Modular transitional implants to support the interim maxillary overdenture. Compend Cont Educ Dent 1999; 20: 975-8.

34. Bichacho N, Landsberg CJ, Rohrer M, Davidovich Y. Immediate fixed transitional restoration in implant dentistry. Pract Periodontics Aesthet Dent 1999; 11: 45-51.

35. Romanos G, Toh CG, Siar CH, Swaminathan D, Ong AH, Donath K, Yaacob H, Nentwig GH. Peri-implant bone reactions to immediately loaded implants. An experimental study in monkeys. J Periodontol 2001, 72: 506-11.

36. Goodacre CJ, Kan KYK, Rungcharassaeng K. Clinical complications of osseointegrated implants. J Prosthet Dent 1999; 81: 537-52.

37. Esposito M, Thomsen P, Ericson LE, Lekholm U. Histopatologic observations on early oral implant failures. Int J Oral Maxillofac Implants 1999; 14: 798-810.
38. McDermott NE, Chuang SK, Woo VV, Dodson TB. Complications of dental implants: identification, frequency, and associated risk factors. Int $\mathrm{J}$ Oral Maxillofac Implants 2003; 18: 848-55.

39. Testori T, del Fabbro M, Feldman S et al. A multicenter prospective evaluation of 2-months loaded Osseotite implants placed in the posterior jaws: 3-year follow-up results. Clin Oral Impl Res 2002; 13: 154-61.

40. Bornstein MM, Lussi A, Schmid B, Belser U, Buser D. Early loading of nonsubmerged titanium implants with a sandblasted and acid-etched (SLA) surface: 3-year results of a prospective study in partially edentulous patients. Int $\mathrm{J}$ Oral Maxillofac Implants 2003; 18: 659-66.

41. Velasco E, Pérez O, Medel R, Segura JJ, Torres R. Carga precoz de los implantes no sumergidos con superficie grabada con ácidos en sobredentaduras mandibulares. Arch Odontoestomatol 2003; 19: 308-16. 